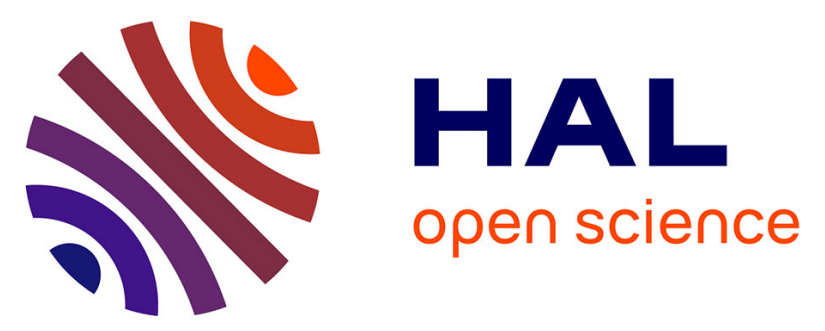

\title{
Prévention de la transition du cancer in situ vers un cancer invasif chez les femmes en surpoids/obèses : rôle des cellules myoépithéliales
}

Habanjar Ola, M. Vermerie, Caroline Decombat, Celine Auxenfans, Mona Diab-Assaf, Florence Caldefie-Chezet, Laetitia Delort

\section{To cite this version:}

Habanjar Ola, M. Vermerie, Caroline Decombat, Celine Auxenfans, Mona Diab-Assaf, et al.. Prévention de la transition du cancer in situ vers un cancer invasif chez les femmes en surpoids/obèses: rôle des cellules myoépithéliales. Journées Francophones de Nutrition, Nov 2021, Lille, France. hal03434890

\author{
HAL Id: hal-03434890 \\ https://hal.science/hal-03434890
}

Submitted on 25 Jan 2022

HAL is a multi-disciplinary open access archive for the deposit and dissemination of scientific research documents, whether they are published or not. The documents may come from teaching and research institutions in France or abroad, or from public or private research centers.
L'archive ouverte pluridisciplinaire HAL, est destinée au dépôt et à la diffusion de documents scientifiques de niveau recherche, publiés ou non, émanant des établissements d'enseignement et de recherche français ou étrangers, des laboratoires publics ou privés. 
Nutrition en pathologie : cancer, insuffisance d'organes, réanimation, pathologies digestive, sarcopénie... JFN2021-ABS-474

\section{Prévention de la transition du cancer in situ vers un cancer invasif chez les femmes en} surpoids/obèses : rôle des cellules myoépithéliales

Ola HABANJAR* ${ }^{*}$, Marion VERMERIE ${ }^{1}$, Caroline DECOMBAT ${ }^{1}$, Celine AUXENFANS ${ }^{2}$, Mona DIAB-ASSAF ${ }^{3}$, Florence CALDEFIE-CHEZET ${ }^{1}$, Laetitia DELORT ${ }^{1}$

${ }^{1}$ Equipe ECREIN, université Clermont-Auvergne, clermont ferrand, ${ }^{2}$ Banque de tissus et cellules, Hôpital EdouardHerriot, Lyon, France, ${ }^{3}$ Equipe Tumorigénèse Pharmacologie moléculaire et anticancéreuse, Faculté des Sciences II, Université libanaise Fanar, beyrouth, Liban

Discipline: Expérimental/mécanismes cellulaires et moléculaires

Présentation préférée: EPOSTER

Introduction et but de l'étude: L'incidence du carcinome canalaire in situ (CCIS) a augmenté au cours des dernières années. Au cours de la transition du CCIS vers un cancer canalaire invasif $(\mathrm{CCl})$, une désorganisation, voire la disparition des cellules myoépithéliales (CME) est observée, renforçant la capacité des cellules cancéreuses à migrer. Aujourd'hui aucun marqueur n'a été défini pour identifier quel CCIS évoluera vers un CCI. Ainsi, la compréhension de la transition des $\mathrm{CCIS}$ vers les $\mathrm{CCl}$ semble être d'une importance majeure pour permettre une meilleure prise en charge des patientes.

De nombreuses études ont montré le rôle du microenvironnement adipeux dans la progression du cancer du sein. Notre équipe a notamment mis en évidence que les cellules adipeuses pourraient être capables de modifier les caractéristiques fonctionnelles des CME conduisant à un processus d'invasion.

Ainsi, notre objectif consiste en l'identification de facteurs préventifs de la transition du CCIS vers un CCI chez les femmes en surpoids ou obèses via l'évaluation de l'impact du microenvironnement adipeux inflammatoire sur les CME en développant des modèles 3D.

Matériel et méthodes: Des modèles 3D seront développés dans ce projet pour évaluer l'impact du microenvironnement adipeux inflammatoire sur les MEC dans le contexte du CCIS.

Un modèle de sphéroïdes reproduisant la pathologie du cancer canalaire in situ est en cours de développement. Pour cela, des cellules de cancers in situ (lignée cellulaire MCF10DCIS) sont d'abord ensemencées dans des moules en agarose ne permettant pas l'adhésion cellulaire. Après obtention de sphéroïdes, des cellules myoépithéliales (lignée cellulaire Hs578Bst) sont ajoutées à notre modèle, l'objectif étant l'obtention d'une couche continue de CME autour des sphéroïdes préalablement formés. Par imagerie confocale, l'organisation des cellules dans les sphéroïdes ainsi que la rupture de la couche continue de CME sera visualisée.

L'impact du microenvironnement adipeux inflammatoire sera ensuite évalué via l'utilisation de milieux conditionnés provenant de la culture de cellules souches adipeuses ou d'adipocytes matures de femmes en surpoids/obèses.

Résultats et Analyse statistique: Une première étape de mise au point du modèle de sphéroïdes a tout d'abord été réalisée. Pour cela, la densité cellulaire ainsi que les durées d'incubation ont été optimisées. La validation des anticorps spécifiques à chaque type cellulaire est en cours de validation par microscopie confocale.

L'étape suivante consistera à évaluer l'impact des cellules adipeuses et de leur sécrétions dans le modèle mis au point. Conclusion: Ce projet pourrait à terme permettre d'agir sur la prévention de l'évolution des cancers canalaires in situ (CCIS) vers les cancers canalaires invasifs $(\mathrm{CCl})$ et conduire au développement de nouvelles stratégies thérapeutiques pour la prise en charge les femmes avec CCIS à haut ou bas risque de développer un CCI.

Conflits d'intérêts: Aucun conflit à déclarer 